\title{
Gellan Gum for Tissue Engineering Applications: A Mini Review
}

\author{
R Spera ${ }^{1 *}$, S Nobile $^{2}$ and L Di Trapani ${ }^{3}$ \\ ${ }^{1}$ Department of Drug Chemistry and Technologies, "Sapienza" University of Rome, Piazzale Aldo Moro 5, 00185 Rome, Italy \\ ${ }^{2}$ Department of Drug Chemistry and Technologies, University of Perugia, Via del Liceo 1, 06123 Perugia, Italy \\ ${ }^{3}$ School of Medicinal Sciences and Health Products, University of Camerino, Via S. Agostino 1, 62032 Camerino, Italy

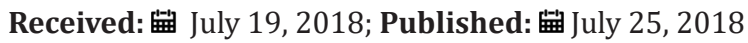

*Corresponding author: R Spera, Department of Drug Chemistry and Technologies, Sapienza University of Rome, Piazzale Aldo Moro 5, 00185 Rome, Italy.

\begin{abstract}
Gellan gum-based hydrogels have been proposed for biomedical applications including bone repair and cartilaginous tissue regeneration. This polysaccharide has the ability to form stable gels without the use of harsh reagents and offers excellent gel stability and flexibility. Gellan gum can function as a minimally invasive injectable system, gelling inside the body in situ under physiological conditions and efficiently adapting to the defect site. This work presents an overview of the recent advances in the research on gellan gum for biomedical applications.
\end{abstract}

Keywords: Gellan Gum; Hydrogel; Cartilage Regeneration

\section{Introduction}

Gellan gum (GG) is a polysaccharide produced by microbial fermentation of the Sphingomonas paucimobilis microorganism. GG is a linear anionic polysaccharide composed of tetrasaccharide (1,3-b-D-glucose, 1,4-b-Dglucuronic acid, 1,4-b-D-glucose, 1,4-a-Lrhamnose) repeating units, containing one carboxyl side group, and was initially described by Moorhouse et al. [1]. This material has a broad use in the food industry and biomedical fields, mostly due to its processing into transparent gels that are resistant to acid stress and exhibit excellent heat resistance up to $120^{\circ} \mathrm{C}$.

It can be dissolved in water, and when heated and mixed with mono or divalent cations, forms a gel upon lowering the temperature under mild conditions. GG exists in two different forms: acetylated and deacetylated. Both form thermo reversible gels, varying in their mechanical properties, in fact the acetylated form produce soft and elastic gels, while the fully deacetylated polysaccharide yields hard and brittle gels.

At high temperatures, GG is in the coil form; upon temperature decrease, a thermally reversible coil to double-helix transition occurs, which is a prerequisite for gel formation. Afterwards, a structure composed of anti-parallel double helices self-assembled to form oriented bundles, called junction zones, is formed. Untwined regions of polysaccharide chains, in the form of extended helical chains, link the junction zones, leading to the formation of a threedimensional network, that creates the gel. The gelation process of GG solutions is strongly influenced by the chemical nature and amount of cations present in the solution. The presence of cations is of paramount importance when a structurally stable gel is to be prepared.
In fact, at low GG concentrations, the helix formation and its partial aggregation may form an ordered structure, but this does not lead to gel formation because the number of helical aggregates does not give rise to a continuous network in the whole volume. The main barrier are the carboxyl side groups that repulse each other by electrostatic interaction, therefore hindering the tight binding of helices and their cohesive aggregation. The introduction of cations shields the electrostatic repulsion and thereby allows the tight binding and aggregation of helices. Furthermore, it is important to note that divalent cations promote the gelation much more strongly than monovalent ones.

GG is commonly used in the food and pharmaceutical industry. However, it has been also proposed as new biomaterial for cartilage tissue engineering applications due to its versatility and efficacy for cartilage repair strategies involving both subchondral stimulation and cell transplantation. GG can be used also as drug delivery carrier, thus highlighting the versatility of this material. In fact, polymeric hydrogels represent ideal candidates for the design of sustained drug release platforms. Their high value relies on the tunable properties, such as swelling, porosity, mechanical stability and responsiveness to physical stimuli. All these factors can be precisely optimized to modulate the loading efficiency and the drug release kinetic.

\section{Review of Recent Research Works}

Agnello et al. [2] synthesized a new GG derivative by covalent attachment of octadecylamine $\left(\mathrm{C}_{18}-\mathrm{NH}_{2}\right)$ to polysaccharide backbone via amide linkage by using bis(4-nitrophenyl) carbonate 
(4-NPBC) as a coupling agent. These new GG derivatives were soluble in deionized water at a temperature lower than starting GG. GG-C ${ }_{18}$ derivative showed a crossover point around physiological temperature, it was possible to obtain hydrogel simply by pouring the polymer solution in media with physiological ionic strength.

Manda etal. [3] GG-hydroxyapatite (HAp) spongy-like hydrogels to mimic the organic (GG) and inorganic (HAp) phases of the bone. HAp was successfully introduced within the GG polymeric networks without compromising the thermostability of the biomaterials. The developed biomaterials showed sustained degradation, high swelling, pore sizes between 200 and $300 \mu \mathrm{m}$, high porosity (>90\%) and interconnectivity $(<60 \%)$ that was inversely proportional to the total polymeric amount and to $\mathrm{CaCl}_{2}$ crosslinker. $\mathrm{CaCl}_{2}$ and HAp reinforced the mechanical properties of the biomaterials from a storage modulus of $40 \mathrm{KPa}$ to $70-80 \mathrm{KPa}$. This study also showed that $\mathrm{HAp}$ and $\mathrm{CaCl}_{2}$ favored the bioactivity and that cells were able to adhere and spread within the biomaterials up to 21 days of culture [4,5]. Overall, the possibility to tailor spongy-like hydrogels properties by including calcium as a crosslinker and by varying the amount of HAp will further contribute to understand how these features influence bone cells performance in vitro and bone formation in vivo.

Pacelli et al. [6] proposed nanocomposite hydrogels are a valid solution to treatment of infected wounds as they can be used as wound dressing materials for the delivery of therapeutic agents at the site of the injury. In addition, their improved mechanical properties make possible a sterile treatment, which is not generally possible using conventional hydrogels. To this aim, gellan gum methacrylate (GG-MA), a biocompatible polymer recently proposed for the fabrication of injectable and photocrosslinkable hydrogels, was combined with laponite to form novel NC hydrogels as innovative wound dressing material.

Razali et al. [7] produced a unique three-dimensional nanostructured GG by incorporating $\mathrm{TiO}_{2}$ nanoparticles by freezedrying. The scaffold possessed good photocatalytic activity after UV irradiation and it induces the cell growth, thus offering a good candidate for biomedical applications.

Vilela et al. [8] proposed GG-MA formulation as a secondgeneration hydrogel for controlled delivery of cartilage-forming cells into focal chondral lesions, allowing immediate in situ retention of cells and 3D filling of lesion volume.

\section{Conclusion}

Interest is growing in the use of hydrogels as bone tissueengineering scaffolds due to advantages such as injectability and ease of incorporation of active substances such as enzymes. GG represents a versatile and promising platform for tissue engineering scaffold due to the possibility to form gels with highly tunable physical and mechanical properties. GG hydrogels can provide support to develop and generate a functional cartilaginous tissue ant to promote bone regeneration. are an important subject of study.

\section{References}

1. Moorhouse R, Colegrove GT, Sandford PA, Baird JK, Kan KS (1981) PS-60: A new gel-forming polysaccharide. Washington DC: American Chemical Society, USA.

2. Agnello S, Gasperini L, Mano JF, Pitarresi G, Palumbo FS, et al. (2017) Synthesis, mechanical and thermal rheological properties of new gellan gum derivatives. International Journal of Biological Macromolecules 98: 646-653.

3. Manda MG, Da Silva LP, Cerqueira MT, Pereira DR, Oliveira MB, et al. (2018) Gellan gum-hydroxyapatite composite spongy-like hydrogels for bone tissue engineering. J Biomed Mater Res Part A 106(2): 479-490.

4. Oliveira JT, Martins L, Picciochi R, Malafaya PB, Sousa RA, et al. (2010) Gellan gum: A new biomaterial for cartilage tissue engineering applications. Journal of Biomedical Materials Research Part A 93(3): 852-863.

5. Pacelli S, Paolicelli P, Avitabile M, Varani G, Di Muzio L, et al. (2018) Design of a tunable nanocomposite double network hydrogel based on gellan gum for drug delivery applications. European Polymer Journal 104: 184-193.

6. Pacelli S, Paolicelli P, Moretti G, Petralito S, Di Giacomo S, et al. (2016) Gellan gum methacrylate and laponite as an innovative nanocomposite hydrogel for biomedical applications. European Polymer Journal 77: 114-123.

7. Razali MH, Ismail NA, Zulkafli MFA, Amin KAM (2018) Nanostructured materials: $\mathrm{TiO}_{2}$ nanoparticles incorporated gellan gum scaffold for photocatalyst and biomedical Applications. Materials Research Express 5: (3).

8. Vilela CA, Correia C, Da Silva A, Tírcia M, Santos C, et al. (2018) In vitro and in vivo performance of methacrylated gellan gum hydrogel formulations for cartilage repair. J Biomed Mater Res Part A 106(7): 1987-1996.

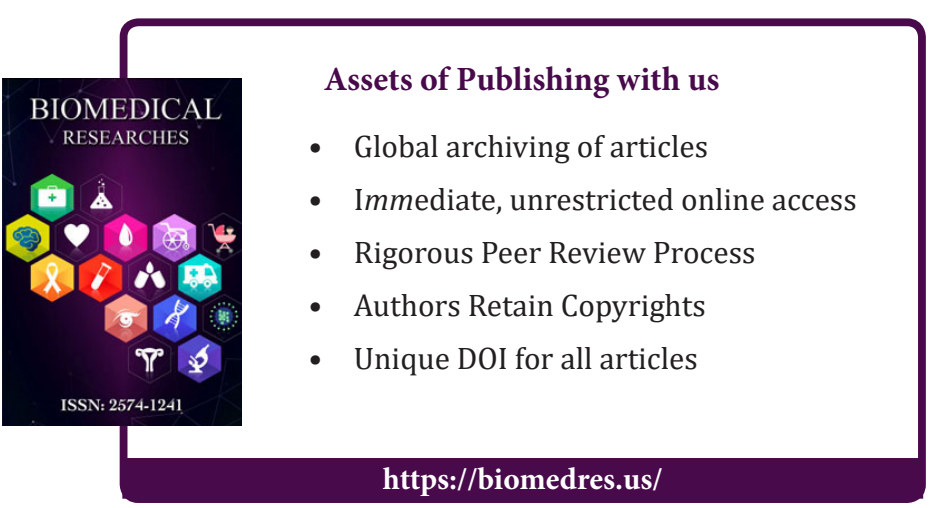

ISSN: 2574-1241

DOI: $10.26717 / B J S T R .2018 .07 .001474$

R Spera. Biomed J Sci \& Tech Res

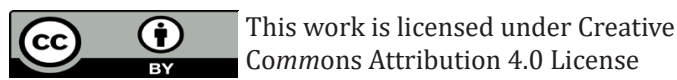

Submission Link: https://biomedres.us/submit-manuscript.php 